https://doi.org/10.48009/4_iis_2019_75-85

Issues in Information Systems

Volume 20, Issue 4, pp. 75-85, 2019

\title{
THE IMPACT OF BEDTIME SMARTPHONE USAGE ON SLEEP HEALTH: A PILOT QUANTITATIVE STUDY
}

\author{
Manvitha Valasareddy, Harrisburg University, mvalasareddy@harrisburgu.edu \\ Wenli Wang, Robert Morris University,wangw@rmu.edu \\ Chaza F. Abdul-Al, Harrisburg University, cabdul@harrisburgu.edu \\ Steven P. Niles, Los Angeles City College, nilessp@lacitycollege.edu
}

\begin{abstract}
With the rising smartphone market penetration, there is also increased health concerns related to smartphone addiction. Bedtime smartphone usage has shown to have adverse effects on sleep by the medical professionals. This research extends and confirms the existing literature. As a pilot quantitative study, it found that all of the respondents bring their smartphones to the bedroom and $83 \%$ of them have smartphones within reach while sleep. Smartphone users tend to sleep late ( $90 \%$ sleep between $10-12 \mathrm{pm}$ ) and have short sleep duration ( $50 \%$ sleep for six hours or less). It also found that marital status moderates bedtime smartphone usage. Two thirds of the respondents (all single but not all married people) would watch television or smartphones after lights off. More than $40 \%$ of the respondents have been awaken up by smartphones at night multiple times. One third of respondents would browse media if waking up at night. 50\% of the respondents perceive that bedtime smartphone usage causes sleep deprivation. $83.3 \%$ of the respondents perceive adverse effects such as restlessness in the morning after high smartphone usage the previous night. This research recommends mindful smartphone usage at bedtime to promote sleep health.
\end{abstract}

Keywords: Health, Technology Use, Smartphone, Sleep

\section{INTRODUCTION}

The usage of electronic devices has increased drastically in the past decade. Most of the people in developed countries own smartphones, tablets, laptops, or computers. Smartphones particularly take the center stage in the markets of electronic devices. Deloitte's global mobile survey in 2018 shows that smartphone's market penetration in the U.S. has arisen from $82 \%$ in 2017 to $85 \%$ in 2018 (Deloitte, 2018).

In comparison, Pew Research Center (2018) reaches a slightly more conservative survey result than that the Deloitte survey and concludes that only $77 \%$ of Americans own smartphones while $95 \%$ own cell phones. However, with regard to the smartphone market penetration by age groups, the Pew 2018 study demonstrates consistent results with those in the Deloitte 2018 survey. It shows high market penetration in younger adults with $94 \%$ of smartphone ownership in age group of $18-29,89 \%$ in age group of 30-49, 73\% in 50-64 and 46\% for 65+, in alignment with Deloitte 2018 survey which shows $94 \%$ penetration of smartphone in age group $18-24,89 \%$ in $25-34,92 \%$ in $35-44,89 \%$ in $45-54$, and $74 \%$ $55+$.

The rising smartphone ownership especially in young age groups also accompanies the increased concerns of smartphone's impact on health. As per 2019 statistics, $66 \%$ of the 2.7 billion worldwide smartphone users are affected by nomophobia (No Mobile Phone phobia) (Deyan, 2019). Studies also show an average user checks the smartphone at least 47 times a day (Deyan, 2019). The addicted behaviors related to smartphones have been studied in psychology and behavioral science (Alhassan et. al., 2018; You et. al. 2019). To extend their results, this research investigates the impact of potential addicted bedtime smartphone usage on sleep.

There are many different reasons for sleep disorders. Bedtime usage of smartphones has become one of the rising reasons for sleep disturbance. Research by the medical professionals has concluded that: 1) the adverse effects of bedtime smartphone usage on sleep health, and 2) the damaged sleep health could further have adverse health effects of obesity, diabetes, and insomnia (Shochat, 2012; Mortazavi \& Mortazavi, 2018; Schweizer et. al., 2016; Hughes \& Burke, 2018; Exelmans \& Van Den Bulck, 2016; Polos et. al., 2015; and Levenson et. al., 2017). 
This research intends to confirm the research results from the medical literature. Moreover, this study further explores demographic moderating factors such as marital status on the effects of smartphone usage before bedtime on sleep health. In addition, with the rising rapid adoption of smartphone in the past few years, it is necessary to check and update if the bedtime smartphone usage has also increased, and to what extent. Furthermore, this study directly asks the respondents to answer if they perceive the causal effects of smartphone usage on their sleep disturbance.

This research specifically asks the following research questions:

Research Question 1: To what extent the bedtime smartphone usage is prevalent, and in what form?

Research Question 2: What are the sleep patterns of smartphone users?

Research Question 3: What demographic factors moderate the impact of the bedtime smartphone usage on sleep?

Research Question 4: What are the perceived impacts of bedtime smartphone usage on sleep?

\section{LITERATURE REVIEW}

Shochat (2012) stated that the usage of electronic media is one of the most important factors affecting sleep in young people and adults. Smartphones with Internet access allow people to browse media and watch videos/movies. Watching videos/movies with screen brightness of the phone makes people more active, which will slowly delay their sleep. Shochat (2012) has concluded that technological advancements have taken their toll on human health with damaging effects on sleep quality, quantity, and timing. Figure 1 is the schematic model of environmental and behavioral factors interfering sleep quality, quantity, and timing, in which technology is regarded as one of the environmental factors (Shochat, 2012).

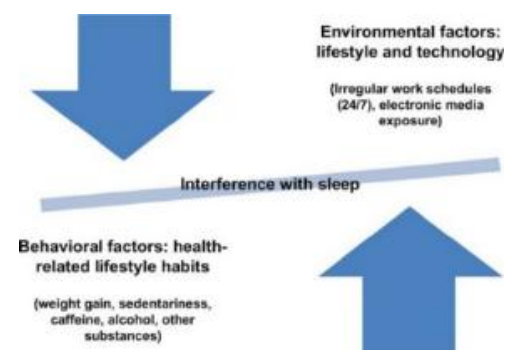

Figure 1. Environmental and behavioral factors interfering sleep quality, quantity, and timing

(Source: Shochat, 2012)

In contrast to Shochat (2012)'s environmental and behavioral concerns related to sleep disturbance, Mortazavi and Mortazavi (2018) explained why smartphone usage at the night-time causes adverse effects on human biologically (see Figure 2). Usually smartphones emit a blue light that suppresses the secretion of melatonin, a sleep hormone. The reduced melatonin secretion disturbs human's circadian rhythm (i.e., the internal body clock for sleep/wake cycle). Mortazavi and Mortazavi (2018) specifically recommended women with hereditary breast cancer avoid using of smart phones at the night-time. When women with hereditary breast cancer uses smartphones or tablets at the night-time, the blue light will amplify their risk of cancer. Hence, it is advised to avoid using smartphones at the night-time or to be used by wearing sunglasses or amber lenses that can reduce the exposure to blue light and hence the risk of disruption in circadian rhythm (Mortazavi and Mortazavi, 2018).

Schweizer et. al. (2016) conducted a longitudinal study among adolescents and showed that ownership of smartphones tends to entail sleep disturbance. They surveyed 591 participants twice with two years apart. They also divided the participants into three groups as owners, non-owners, and new owners. They found all three groups decreased their sleeping time between time 0 and time 1 . At time 0 (with average age around 14), owners of a smartphone were found to sleep significantly less than non-owners or new-owners especially on school days, and to report significantly more sleeping problems. At time 1, owners and new-owners showed no significant differences on sleep duration or sleeping problems. 
Hughes and Burke (2018) measured the impact of refraining from overnight smartphone use on wellbeing. They asked the experimental group participants who abstained from smartphone use in the bedroom for one week to answer the four surveys pre- and post the experiment: Subjective Happiness Scale, Quality of Life Scale, Smartphone Addiction Scale, and Intensity \& Time Affect Survey. In three out of these four measures, the hypothesis of increased wellbeing was upheld.

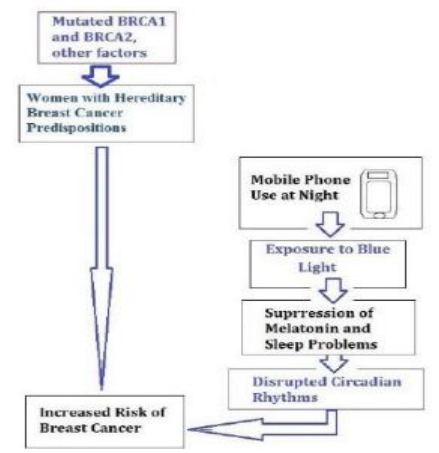

Figure 2. Impact of night-time smartphone usage on breast cancer (Source: Mortazavi \& Mortazavi, 2018)

Exelmans and Van Den Bulck (2016) studied the bedtime use of mobile phone and sleep. They concluded that $50 \%$ of the respondents owned a smartphone, and $60 \%$ took their mobile phones to the bedroom. Calling/texting after lights out have significantly predicted respondents' scores on the Pittsburgh Sleep Quality Index (PSQI), particularly "longer sleep latency, worse sleep efficiency, more sleep disturbance, and more daytime dysfunction." Using the Fatigue Assessment Scale (FAS) and the Bergen Insomnia Scale (BIS), they found that bedtime mobile phone use predicted respondents' self-reported later rise time, higher insomnia score, and increased fatigue.

Exelmans and Van Den Bulck (2016) also found that age significantly moderated the relationship between bedtime mobile phone use and fatigue, rise time, and sleep duration: increased bedtime mobile phone usage was associated with more fatigue and later rise times among younger respondents ( $\leq$ around 40 years old); but it was related to an earlier rise time ( $\geq 60.15$ years old) and shorter sleep duration ( $\geq 66.4$ years old) among older respondents.

Polos et al. (2015) examined the extent and impact of mobile device-based Sleep Time-Related Information and Communication Technology (STRICT) use among 3139 U.S. adolescents (average age of 13.3 years) and found that about $62 \%$ of them used STRICT after bedtime, $56.7 \%$ texted/tweeted/messaged in bed, and $20.8 \%$ awoke to texts. They also found that STRICT use was associated with insomnia, daytime sleepiness, eveningness, academic underperformance, later bedtimes, and shorter sleep duration.

Levenson, et al. (2017) conducted a large-scale study of 1763 young adults (19 - 32 years) in the U.S. and found that using social media within thirty minutes before bed had an odd ratio of 1.62 for increased sleep disturbance. Importantly, the more frequently the young adults checked their social media before bed, the greater their sleep disturbances were likely to be.

\section{RESEARCH METHODOLOGY}

A questionnaire was designed to seek answers to the aforementioned four research questions. Google form was used to collect survey responses. Table 1 provides a summary of the sixteen (16) survey questions in four categories: demographics, smart device usage, the usage of smart devices before bedtime, and the impact of smartphone usage on general health and sleep health particularly. 
Table 1. Survey Instrument

\begin{tabular}{|l|l|}
\hline \multirow{4}{*}{ Demographics (3) } & Gender \\
\cline { 2 - 2 } & Age group \\
\cline { 2 - 2 } & Marital Status \\
\hline \multirow{4}{*}{ Smart device usage (3) } & Which smart devices do you have /use most? \\
\cline { 2 - 2 } & How many hours do you use smartphone in a day? \\
\cline { 2 - 2 } & For what purpose do you use the smartphone most? \\
\hline \multirow{5}{*}{$\begin{array}{l}\text { Usage of bedtime smart } \\
\text { devices (4) }\end{array}$} & Ohat is your regular bedtime? \\
\cline { 2 - 2 } & Do you have any health ailments caused by sleep deprivation? \\
\cline { 2 - 2 } & Do you have smart devices in your bedroom? \\
\cline { 2 - 2 } & Where do you keep your smartphone while you sleep? \\
\cline { 2 - 2 } & How often have you been awakened by your smartphone at night? \\
\cline { 2 - 2 } $\begin{array}{l}\text { Impact of bedtime } \\
\text { smartphone usage on } \\
\text { general health and sleep } \\
\text { health particularly (3) }\end{array}$ & What will you do if you wake up in the middle of sleep? \\
\cline { 2 - 2 } & Do what extent do you perceive accessibility via smartphones as stressful? \\
\cline { 2 - 2 } & $\begin{array}{l}\text { Dofter highly using smartphone the previous night, what kind of observations } \\
\text { do you see the next morning? (e.g., Restless, irritated, etc.) }\end{array}$ \\
\hline
\end{tabular}

Demographic information on gender, age group, marital status was collected. The survey specifically targeted the age group of 25 to 34 years old respondents. Emails were sent out to the respondents who were supposed to belong to this age group. The reason of focusing on this age group was due to the result of the Deloitte 2018 survey, which has shown that this particular age group has used phones for almost all of the online activities (including check bank balances and online search) except of making online purchases (prefer laptop) or playing video games (prefer game console). In comparison, all the other age groups have preferred laptop, desktop, or tablets for the activities such as online search or check bank balances. Figure 3 shows the Deloitte 2018's survey results on "preferred devices for online activities" (Deloitte, 2018).

The marital status was collected because the research is about the smartphone usage before bed. Marital status may affect the smartphone usage because married couples typically share the same bedroom and singles typically sleep alone.

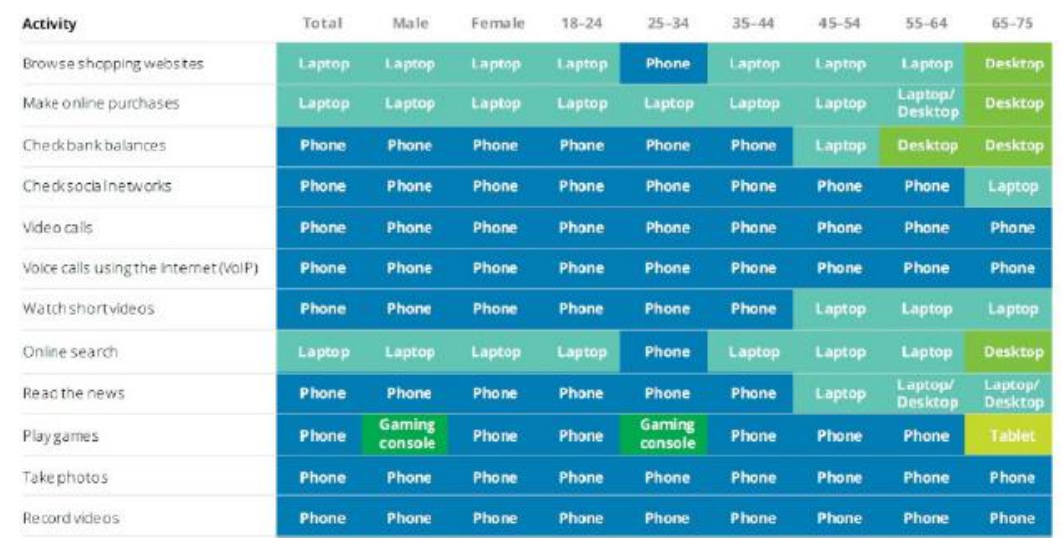

Figure 3. Preferred devices for online activities (Source: Deloitte 2018) 


\section{RESULTS}

The survey was issued mostly through the word of mouth. The administration of the survey took place in Spring 2019. Since it is a pilot study, the survey was given only to limited number of respondents. Twelve (12) survey respondents answered the survey.

\section{Demographics}

This survey targeted mostly young adults. The results reflected this targeted demographic group. Among the twelve (12) survey participants, ten (10) (75\%) out of them were between the age of 25 and 34, two (2) (16.7\%) were between 35 and 44, and one (1) (8.3\%) fell into the age range of 45-60 (see Figure 4).

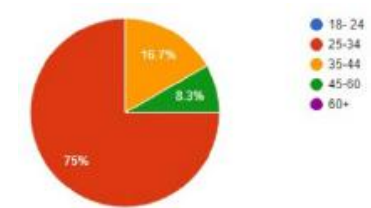

Figure 4. Demographics: Age

There were seven (7) male respondents and five (5) female ones. The percentages of respondents were $56.3 \%$ male and $41.6 \%$ female (see Figure 5). The male to female ratio was roughly in alignment with the male to female ratio in smartphone usage in the Deloitte 2018 survey (Deloitte, 2018).

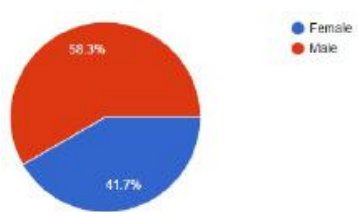

Figure 5. Demographics: Gender

The results in marital status just happened to be favorable to the analysis. $50 \%$ of the respondents were married and $50 \%$ were single (see Figure 6). This fortunate 50-50 split was favorable to do group comparison based on this particular demographic data.

\section{Smart Device Usage}

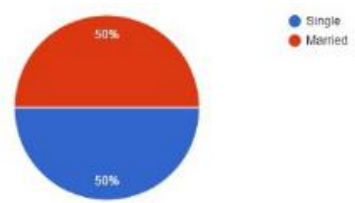

Figure 7 shows the smart devices owned by the survey respondents. All of them (100\%) owned smartphones. $75 \%$ owned computers/laptops, $58.3 \%$ owned televisions, and $25 \%$ owned tablet or iPad. Figure 7 also shows the ranking of the most usage by smart devices in the order of smartphone, tablet/iPad, television, and computer/laptop. Smartphone ranked the highest among all (100\%) respondents for the most usage. Computers/laptops ranked the lowest among all $75 \%$ of the respondents who owned them. Tablet/iPad ranked the second most used smart devices for all $25 \%$ of the respondents who owned them. All of these $25 \%$ respondents also owned televisions but they ranked higher usage of tablet/iPad than the use of televisions. In other words, television usage ranked either as the second for those without tablet/iPad ownership or the third for those with tablet/iPad ownership.

Figure 8 shows the number of hours of daily smartphone usage. $33.3 \%$ of the respondents have more than five hours of daily smartphone usage. $41.7 \%$ have 3 to 5 hours, $16.7 \%$ have 3 hours, and $8.3 \%$ has 1 hour of daily smartphone usage. 


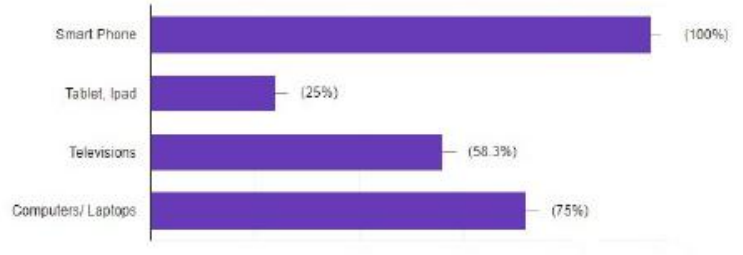

Figure 7. Smart devices owned and ranked by most usage

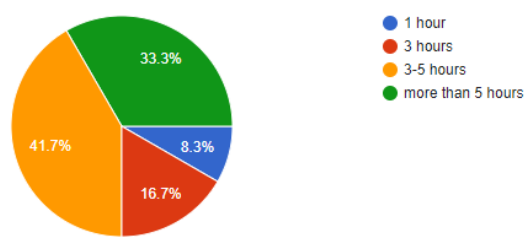

Figure 8. Number of hours of daily smartphone usage

Figure 9 shows the main purpose of using smartphone the most. 50\% of the respondents ranked "calls/texting" as the main purpose of smartphone usage. 33.3\% ranked "watch videos/movies," and $16.7 \%$ ranked "browsing/emails" as the main purpose. None has ranked "playing games" as the main purpose.

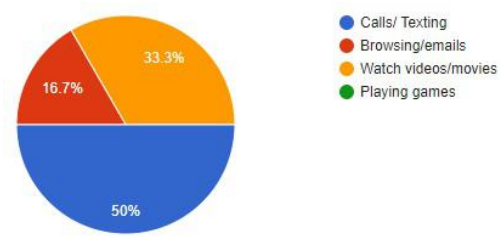

Figure 9. Main Purpose of daily smartphone usage

\section{Sleep Patterns}

Figure 10 shows the regular bedtime of respondents. $50 \%$ of them go to bed between $11 \mathrm{pm}$ to $12 \mathrm{pm}, 41.7 \%$ between $10-11 \mathrm{pm}$. $8.3 \%$ between $8-10 \mathrm{pm}$. None goes to bed between $7-8 \mathrm{pm}$.

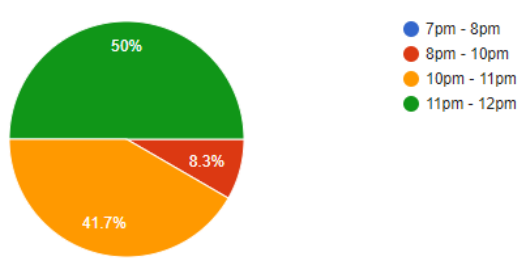

Figure 10. Regular bedtime

Figure 11 shows the average sleep hours. The majority (41.7\%) has an average of six hours of sleep. $25 \%$ has an average of eight hours sleep. $16.7 \%$ has an average of seven hours of sleep. $8.3 \%$ has 10 hours of sleep and $8.3 \%$ only sleeps for 5 hours on average. 


\section{Issues in Information Systems}

Volume 20, Issue 4, pp. 75-85, 2019

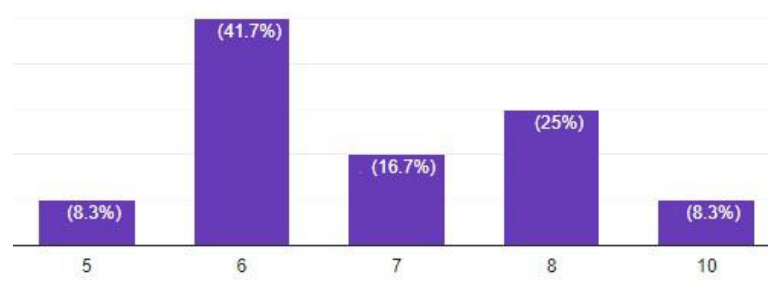

Figure 11. Average sleep hours

Figure 12 shows the percentage of respondents' awareness if they have any health ailments caused by sleep deprivation. The majority of them ( $83.3 \%$ ) do not have health ailment caused by sleep deprivation, $8.3 \%$ has such a health ailment, and $8.3 \%$ responded "I don't know." The respondent with health ailments caused by sleep deprivation was also the one who on average has 10 hours of sleep.

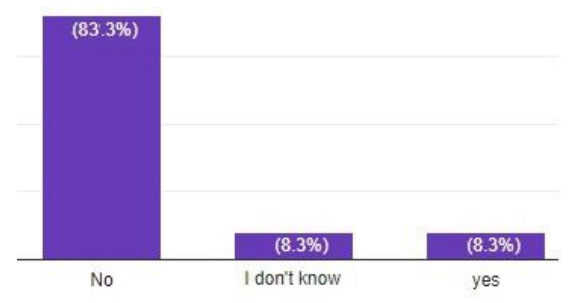

Figure 12. Any health ailment caused by sleep deprivation

\section{Usage of bedtime smart devices}

Figure 13 shows $100 \%$ of respondents have smart devices in the bedroom.

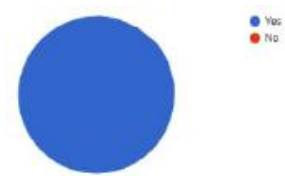

Figure 13. Smart devices in bedroom

Figure 14 shows that $16.7 \%$ of the respondents keep their smartphones in bedroom but out of reach while sleep. The rest (83.3\%) have their smartphones in bedroom and within reach while sleep. For the respondents who have their smartphones out of reach in bedroom, they have an average seven hours of sleep, are between age 25 and 34 , with bedtime of 11-12pm, only own smartphones as smart devices, have 3-5 hour of daily smartphone usage, and use smartphone mostly for "calling/texting."

Figure 15 shows that $66.7 \%$ of the respondents you watch television or smartphone after lights are turned off. $25 \%$ do not watch television or smartphone and $8.3 \%$ chose "maybe" after lights are turned off. For those whose answers were "No" or "Maybe," they are all married. In other words, 75\% of married respondents do not watch television or smartphone after lights are off. All respondents who are single answered "Yes," which means they all watch TV or smartphone after light are turned off. 


\section{Issues in Information Systems}

Volume 20, Issue 4, pp. 75-85, 2019

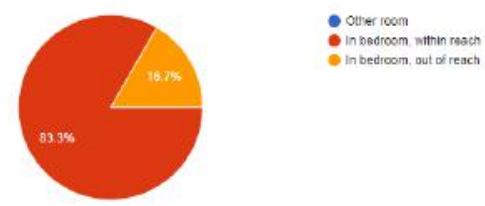

Figure 14. Smartphones in bedroom and within reach while sleep
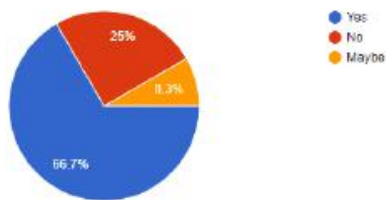

Figure 15. Watching TV or smartphones after lights turned off

Figure 16 shows that $58.3 \%$ of the respondents have never got awaken by their smartphones at night. $33.3 \%$ have been awaken by smartphones at night multiple times. $8.3 \%$ of the respondents have been awaken by smartphone almost everyday. This particular respondent uses smartphone more than five hours a day, is one of the two respondents whose main purpose of smartphone use is to "browsing/email," is married, answered "maybe" to watch television or smartphone when lights are off, goes to bed between $10-11 \mathrm{pm}$, and is in the age group of $35-44$, and male.

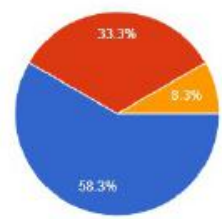

- Never

- Mutíle times

Figure 16. Frequency of being awaken by smartphone at night

Figure 17 shows that $66.7 \%$ of the respondents will try to sleep again after waking up in the middle of sleep. But $33.3 \%$ will try to browse some media. $75 \%$ of those who would browse some media are singles with various number of hours of daily smartphone usage. $75 \%$ of them are males.

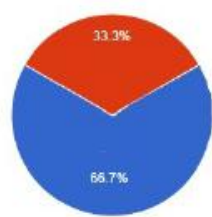

- Ty to sleed acain - Do some work

Figure 17. Activities to do after waking up in the middle of sleep

\section{Impact of bedtime smartphone usage on general health and sleep health particularly}

Figure 18 shows that $50 \%$ of the respondents feel "not at all stressful" from smartphone use. $41.7 \%$ feel "little stressful" and $8.3 \%$ feels "very stressful" from smartphone use. The respondent feels very stressful of smartphone use is a female, single, in age group of 25-34, who has smartphone in bedroom and within reach while sleep, wakes up by smartphone multiple times at night, uses smartphone mainly for "calling/texting," has an average 3-5 hour of daily smartphone usage, but does not have any health ailments caused by sleep deprivation. 


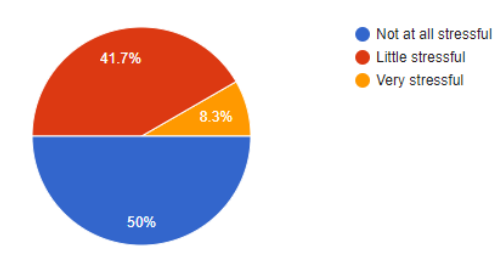

Figure 18. Stress level from smartphone use

Figure 19 shows that $50 \%$ of the respondents regard the smartphone use as the cause of sleep deprivation. $33.3 \%$ hold the opposite view. 8.3\% answered "maybe" and 8.3\% answered "Don't know." Among the $50 \%$ of respondents who answered "yes" to the smartphone usage causing sleep deprivation, $66.7 \%$ of them are married. The respondent who has felt "very stressful" from smartphone usage also regards the smartphone use has caused sleep deprivation. Among the $33.3 \%$ holding the opposite view, $75 \%$ of them are single.

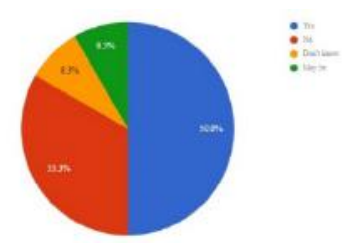

Figure 19. Smartphone use is the cause for sleep deprivation

When asked about "After highly using smartphone the previous night, what kind of observations do you see the next morning? (e.g., restless, irritated, etc.)," $50 \%$ of respondents felt "restless," $8.3 \%$ felt "headache and irritated," $8.3 \%$ added "tired" besides "restless," 8.3\% felt "agitated," $8.3 \%$ felt "sleepless," and $8.3 \%$ felt "eyes burn." Only $8.3 \%$ felt "normal" and $8.3 \%$ felt "none." Overall, $83.3 \%$ of the respondents have felt adverse effects in the morning after the high smartphone activities the previous night. The $16.7 \%$ of the respondents have not felt adverse effects and they are both male in the age group of 25-34, keep their smartphone in bedroom within reach while sleep, have never been awaken by smartphone at night, and feel no stress from smartphone use. One of them has an average 10 hours of sleep and does have health ailments caused by sleep deprivation, has more than 5 hours of daily smartphone usage and uses the smartphone mainly for "browsing/emails."

\section{DISCUSSIONS}

Smartphone usage is prevalent. $75 \%$ of the respondents spend more than three hours in daily smartphone use. $100 \%$ of the respondents have smart devices in bedroom, and $83.3 \%$ of them have smartphone in bedroom within reach while at sleep, which indicates the wide-spread dependency (if not addiction yet) to smartphones. The main purpose of smartphone usage for $50 \%$ of the respondents is for calling and texting, while for one third of the respondents is for watching videos/movies. Two thirds of the respondents would watch television or smartphones after lights are turned off in the bedroom. Married people have different use pattern of smartphones in bedroom before bedtime from that of single people. All single people would watch television or smartphones after lights-off while married people would not. Above $40 \%$ of the respondents have been awaken up by smartphones at night multiple times. One third of respondents would browse media if waking up at night.

People also sleep late in general. More than $90 \%$ of the respondents' bedtime is between $10-12 \mathrm{pm}$. The average sleep time is short than the recommended eight hours of sleep; $50 \%$ of the respondents sleep for six hours or less while only $25 \%$ of respondents sleep for eight hours. Although respondent did not regard smartphone use causing stress, $50 \%$ of them regard smartphone use causing sleep deprivation. $83.3 \%$ of the respondents have felt adverse effects in the morning after the high smartphone activities the previous night. The adverse effects include restlessness, irritation, agitation, tiredness, sleeplessness, and eye burn. Those who responded no adverse effects were male in the age group of 25-34.

This research has found that the respondents perceive adverse impact of smartphone usage on sleep health, although inclusive on the general stress levels. The result of this research is in alignment with the literature. Increased use of 
smartphones before the bedtime in the bedroom may cause delayed bedtime, shorter sleep time, and more interrupted sleep at night.

Healthy sleep habits are critical for general health. Poor sleep habits lead to reduced cognitive thinking, weight gain, depression, fast aging, forgetfulness, and even increased risk of death. Sleep deprivation may increase irritability, reduce the ability to focus which may even lead to tragic accidents. Hence, reducing the risks affiliated with sleep deprivation is important. One way to reduce such risks is to reduce the use of smartphones before bedtime or even to completely restrict the smartphone from bedrooms. If smartphone needs to be in the bedroom for emergency concerns, it is recommended to turn off other smart devices except for the smartphone, set smartphones only for calls and texts, and keep the smartphones out of reach during sleep.

Due to time constraints, this research is only a pilot quantitative study. Since the survey questions directly ask its respondents of their views on the causal effects of smartphone usage on sleep health, the small sample size still leads to some interesting and meaningful results. For instance, the marital status of the respondents plays an important role in smartphone usage patterns in bedroom before bedtime. This is probably due to the behavioral differences between married couples and single persons in bedrooms before bedtime, and/or the shared experiences of married couples in smartphone usage. Future research will take into account of these behavioral differences and may survey married couples as paired respondents. Gender also plays a role in smartphone usage and sleep health. This would be explored further as well. It would also be interesting to study what smartphone usage patterns in bedroom and before bedtime fall under the nomophobia category, which may require professional interventions for potential addiction behaviors.

\section{CONCLUSIONS}

This research studied and confirmed the adverse effects of bedtime smartphone usage on sleep health. Smart device users should be more mindful about the potential adverse effects of excessive smartphone usage on health. Having a good sleep is an important component of general health. Smartphone is a tool, which can be used to either promote or impede health. This research recommends the reduction of smartphone usage before bedtime in order to improve healthy sleep behaviors. In addition, it suggests mindful smartphone usage by enhancing the situational awareness of time and place of the smartphone usage in order to promote rather than impede health.

\section{REFERENCES}

Alhassan, A. A., Alqadhib, E. M., Taha, N. W., Alahmari, R. A., Salam, M., \& Almutairi, A. F. (2018). The relationship between addiction to smartphone usage and depression among adults: a cross sectional study. BMC Psychiatry, 18(1), 148.

Deyan G. (2019). 45 Smartphone Addiction Statistics 2019. Retrieved from https://techjury.net/statsabout/smartphone-addiction/

Deyan G. (2019). 60+ Smartphone Statistics in 2019. Retrieved from https://techjury.net/stats-about/smartphoneusage/

Deloitte (2018). 2018 Global mobile consumer survey: US edition - A new era in mobile continues. Retrieved from https://www2.deloitte.com/content/dam/Deloitte/us/Documents/technology-media-telecommunications/ustmt-global-mobile-consumer-survey-exec-summary-2018.pdf

Exelmans, L., \& Van Den Bulck, J. (2016). Bedtime mobile phone use and sleep in adults. Social Science \& Medicine, 148, 93-101.

Hughes, N., \& Burke, J. (2018). Sleeping with the frenemy: How restricting 'bedroom use' of smartphones impacts happiness and wellbeing. Computers in Human Behavior, 85, 236-244. 
Levenson, J., Shensa, A., Sidani, J., Colditz, J., \& Primack, B. (2017). Social media use before bed and sleep disturbance among young adults in the United States: A nationally representative study. Sleep, 40(9).

Mortazavi, S. A. R., \& Mortazavi, S. M. J. (2018). Women with hereditary breast cancer predispositions should avoid using their smartphones, tablets and laptops at night. Iranian Journal of Basic Medical Sciences, 21(2), 112-115.

Pew Research Center (2018). Mobile fact sheet. Retrieved from https://www.pewinternet.org/fact-sheet/mobile/.

Polos, P. G., Bhat, S., Gupta, D., O’Malley, R. J., Debari, V. A., Upadhyay, H., Chaudhry, S., Nimma, A., PintoZipp, G., \& Chokroverty, S. (2015). The impact of Sleep Time-Related Information and Communication Technology (STRICT) on sleep patterns and daytime functioning in American adolescents. Journal of Adolescence, 44, 232-244.

Schweizer, A., Berchtold, A., Barrense-Dias, Y., Akre, C., \& Suris, J. (2017). Adolescents with a smartphone sleep less than their peers. European Journal of Pediatrics, 176(1), 131-136.

Shochat T. (2012). Impact of lifestyle and technology developments on sleep. Nature and Science of Sleep, 4, 19-31.

You, Z., Zhang, Y., Zhang, L., Xu, Y., \& Chen, X. (2019). How does self-esteem affect mobile phone addiction? The mediating role of social anxiety and interpersonal sensitivity. Psychiatry Research, 271, 526-531. 